\title{
Intraoperative Pathologic Evaluation with Targeted Axillary Dissection
}

\author{
Editorial for "Intraoperative Touch Imprint Cytology in Targeted Axillary Dissection \\ After Neoadjuvant Chemotherapy Among Breast Cancer Patients with Initial Axillary \\ Metastasis"
}

\author{
Abigail S. Caudle \\ Department of Breast Surgical Oncology, University of Texas MD Anderson Cancer Center, Houston, TX
}

The management of axillary disease in breast cancer patients is constantly evolving; however, the practice of routine axillary clearance for clinically node-positive patients has recently been questioned. With the increased use of neoadjuvant chemotherapy (NAC), as well as increasing efficacy of systemic therapy, a substantial proportion of clinically node-positive patients may achieve a nodal pathologic complete response (pCR) with chemotherapy. Several recent studies have explored minimally invasive techniques to identify those patients who may not benefit from extensive axillary surgery. ${ }^{1-4}$ One such approach is targeted axillary dissection (TAD). ${ }^{4,5}$ With this technique, lymph nodes with biopsy-confirmed metastases are marked with a clip prior to initiating NAC. TAD involves selective localization and removal of the clip, in addition to removal of the sentinel nodes for axillary staging. Wu et al. report their results of using TAD and the use of intraoperative touch imprint cytology to evaluate the excised lymph nodes. They prospectively enrolled patients with biopsy-confirmed nodal metastases who underwent NAC followed by TAD and completion axillary lymph node dissection (ALND). They used intraoperative touch imprint cytology to assess nodes removed by TAD and compared this with the final pathologic results, finding a sensitivity of $87.9 \%$ and specificity of $94.9 \%$. The authors

(C) Society of Surgical Oncology 2018

First Received: 12 June 2018;

Published Online: 27 July 2018

A. S. Caudle

e-mail: ascaudle@mdanderson.org found that a false negative result for intraoperative analysis was more likely when only micrometastatic disease remained.

The authors should be congratulated for this contribution to the field. While the use of intraoperative analysis of sentinel lymph nodes (SLNs) has been found to be an accurate, cost-effective approach in treatment-naive patients, ${ }^{7,8}$ we cannot assume that the evaluation will be as accurate after therapy. Luckily, the authors of that article show that touch imprint cytology correctly identified the presence of metastases in 33 of the 37 cases with confirmed residual disease. A recent study from Memorial Sloan Kettering evaluating frozen section analysis of SLNs after NAC also found that this approach to intraoperative evaluation was beneficial, finding a false negative rate (FNR) of only $6.2 \% .^{9}$ These studies support the use of intraoperative analysis in this setting as the decision to proceed with ALND can be made in a single operative setting. This spares patients the anxiety and risks associated with multiple operations, as well as the delays in starting adjuvant radiation.

The authors describe the limitations of intraoperative assessment. First, small-volume disease such as micrometastases and isolated tumor cells (ITCs) may be missed. In their report, all false negative (FN) results for touch imprint cytology were due to the small volume of residual disease. This was also seen in the article by Moo et al. ${ }^{9}$ in which $30 \%$ of the 33 false negative results had ITCs on final pathology and $46 \%$ had micrometastasis. There is growing recognition that this small-volume disease is relevant in patients after NAC. The American College of Surgeons Oncology Group (ACOSOG) Z1017 trial and the Sentinel Node Biopsy Following Neoadjuvant 
Chemotherapy (SN FNAC) trial showed that the use of immunohistochemistry reduces the FNR of SLN dissection (SLND). ${ }^{2,10}$ One concern is that the authors reported three false positive results, of which two occurred due to misclassification of macrophages and lymphocytes as tumor cells, and one was due to treatment effect seen in the node. Treatment effect can result in fibrosis, mucin, or groups of foamy histiocytes, which makes evaluation of nodes after NAC more challenging than when nodes are retrieved prior to therapy. ${ }^{11}$ Pathologists and surgeons must discuss the intraoperative findings and place them in clinical context. If the diagnosis of a positive node is in doubt, the decision on whether to perform a completion ALND should be based on clinical judgment, or deferred until final pathology is available.

Specific examination of nodes that had biopsy-confirmed metastases at the time of diagnosis is a logical addition to staging of the axilla after NAC. In the ACOSOG Z1071 trial, the FNR of SLN was 6.8\% if the clipped node was retrieved as an SLN. ${ }^{1,12}$ In a study from our institution, the FNR of evaluating the clipped node alone was $4.2 \%$, which dropped to $2 \%$ when TAD was performed. ${ }^{4}$ Recognizing the advantage of this, the current National Comprehensive Cancer Network (NCCN) guidelines recommend routine clipping of nodes with biopsyconfirmed disease, with removal at the time of surgery. ${ }^{13} \mathrm{~A}$ recent survey of members of the American Society of Breast surgeons revealed that $67 \%$ now place clips in nodes at the time of biopsy, and $71 \%$ of those localize them either with a wire (73\%), seed (13\%), or other technique $(14 \%)$. In those who do not localize the clipped node, $82 \%$ perform an X-ray to confirm that the clipped node was removed for evaluation. ${ }^{14}$

The benefit of localizing the clipped node is that it is not always retrieved as an SLN. Thus, relying on SLN alone in these cases would miss evaluation of the node known to have cancer prior to NAC. Wu et al. ${ }^{6}$ report that this was the case in $67 \%$ of their patients. In the ACOSOG Z1071 trial, the clipped node was an SLN in $63 \%$ of cases, and was definitely not an SLN in $20 \%$ of cases. ${ }^{12}$ In the remaining 20\%, it is unknown whether the SLN specimens contained the clipped node or not. This was also seen in our data where the clipped node was not an SLN in $23 \%$ of cases. ${ }^{4}$ Similarly, a study from the University of Pittsburgh reported a rate of $27 \% .^{15}$

Several institutions have published on their experience with localizing clipped nodes. ${ }^{15-19}$ One group from Spain reports an FNR for evaluation of the clipped node alone of $4.1 \%$, similar to our institution's observed FNR of 4.2\%. ${ }^{4,18}$ A group from The Netherlands has reported an FNR of $7 \%$ for their marking the axillary lymph node with radioactive seed (MARI) procedure, where the seed is placed at the time of the original biopsy and left in place through NAC. ${ }^{17}$ Less data are currently available regarding the FNR of TAD, which adds the assessment of SLNs to the clipped node. Diego et al. reported no false negative results in 11 patients. ${ }^{15} \mathrm{We}$ anticipate that the group from the Mayo Clinic, which has reported the technical results of their first experiences with the technique, will report their outcomes in the future, ${ }^{19}$ as will the Dutch group, which is currently conducting a prospective trial. ${ }^{20}$ The authors in this paper report an FNR of $10.8 \%$, which is higher than our initial experience with this technique. ${ }^{4}$ While the authors do not elaborate on this group, we have identified factors that contribute to increased FNRs that could have been in play. ${ }^{15}$ First, similar to ACOSOG Z1071, SENTINA, and SN FNAC, we also found that the use of the dual tracer technique impacts accuracy. ${ }^{1-3}$ In data presented at the Society of Surgical Oncology (SSO) annual meeting, ${ }^{21}$ use of the single tracer technique resulted in an FNR of 5.6\% (95\% confidence interval [CI] 0.7-13), compared with $1.1 \%$ (95\% CI $0.03-6$ ) with dual tracers. The second factor that might contribute is the number of abnormal nodes seen on initial imaging. In the same SSO presentation, we reported that the FNR was $1.1 \%$ (95\% CI 0.03-5.9) if less than four abnormal nodes were seen on the first ultrasound, compared with 5.7\% (95\% CI 0.7-19.2) if four or more nodes were seen. As this practice increases, more reports should be available describing other institutions' experience with TAD, which will allow us to assess the technique in a variety of settings.

Adding intraoperative evaluation of nodes retrieved in TAD has the potential to benefit patients if it is reliable, which this article supports. Omission of ALND if no residual metastasis is seen is somewhat controversial since data reporting oncologic outcomes when completion ALND is not performed are not currently available. However, $85 \%$ of respondents in the American Society of Breast Surgeons' survey said they offer SLND to at least some of their patients, suggesting that many surgeons are beginning to change their practice. ${ }^{14}$ The ability to assess nodal response intraoperatively will help in this transition as, in the current guidelines, patients with a positive node after chemotherapy require axillary clearance. The Alliance A011202 trial, which randomizes clinically nodepositive patients who have a positive SLN after chemotherapy to ALND with nodal radiation versus nodal radiation alone, is currently in accrual and may affect this paradigm in the future.

\section{REFERENCES}

1. Boughey J, Suman V, Mittendorf E, et al. Sentinel lymph node surgery after neoadjuvant chemotherapy in patients with nodepositive breast cancer: the ACOSOG Z1071 (Alliance) clinical trial. JAMA. 2013;310(14):1455-1461. 
2. Boileau JF, Poirier B, Basik M, et al. Sentinel node biopsy after neoadjuvant chemotherapy in biopsy-proven node-positive breast cancer: The SN FNAC study. J Clin Oncol. 2015;33(3):258-264.

3. Kuehn T, Bauerfeind I, Fehm T, et al. Sentinel-lymph-node biopsy in patients with breast cancer before and after neoadjuvant chemotherapy (SENTINA): a prospective, multicentre cohort study. Lancet Oncol. 2013;14(7):609-618.

4. Caudle A, Yang W, Krishnamurthy S, et al. Improved axillary evaluation following neoadjuvant therapy for patients with nodepositive breast cancer using selective evaluation of clipped nodes: implementation of targeted axillary dissection. J Clin Oncol. 2016;34(10):1072-1078

5. Caudle A, Yang W, Mittendorf E, et al. Selective surgical localization of axillary lymph nodes containing metastases in patients with breast cancer: a prospective feasibility trial. JAMA Surg. 2015;150(2):137-43.

6. Wu S, Wang Y, Zhang N, et al. Intraoperative touch imprint cytology in taregeted axillary dissection after neoadjuvant chemotherapy among breast cancer patients with initial axillary metastasis. Ann Surg Oncol. 2018.

7. Jeruss J, Hunt $\mathrm{K}$, Xing $\mathrm{Y}$, et al. Is intraoperative touch imprint cytology of sentinel lymph nodes in patients with breast cancer cost effective? Cancer. 2006;107(10):2328-2336.

8. Lee A, Krishnamurthy S, Sahin A, Symmans W, Hunt K, Sneige $\mathrm{N}$. Intraoperative touch imprint of sentinel lymph nodes in breast carcinoma patients. Cancer. 2002;96(4):225-231.

9. Moo T, Edelweiss M, Hajiyeva S, et al. Is low-volume disease in the sentinel node after neoadjuvant chemotherapy an indication for axillary dissection?? Ann Surg Oncol. 2018;25(6):1488-1494.

10. Boughey J, Ballman K, Symmans W, et al. Methods impacting the false negative rate of sentinel lymph node surgery in patients presenting with node positive breast cancer (T0-T4,N1-2) who receive neoadjuvant chemotherapy: results from a prospective trial-ACOSOG Z1071 (Alliance) [poster]. San Antonio Breast Cancer Symposium 2014. http://eposter.abstractsonline.com/sabc s. Accessed 31 Jan 2015.

11. Provenzano E, Bossuyt V, Viale G, et al. Standardization of pathologic evaluation and reporting of postneoadjuvant specimens in clinical trials of breast cancer: recommendations from an international working group. Mod Pathol. 2015;28(9):1185-1201.

12. Boughey J, Ballman K, Le-Petross $\mathrm{H}$, et al. Identification and resection of clipped node decreases the false-negative rate of sentinel lymph node surgery in patients presenting with nodepositive breast cancer (T0-T4, N1-N2) who receive neoadjuvant chemotherapy: results from ACOSOG Z1071 (Alliance). Ann Surg. 2016;263(4):802-807.

13. National Comprehensive Cancer Network. NCCN clinical practice guidelines in oncology_-breast cancer. 2018;1.2018. www. nccn.org. Accessed 10 Jun 2018.

14. Caudle A, Bedrosian I, Milton D, et al. Use of sentinel lymph node dissection after neoadjuvant chemotherapy in patients with node-positive breast cancer at diagnosis: practice patterns of american society of breast surgeons members. Ann Surg Oncol. 2017;24(10):2925-2934.

15. Diego E, McAuliffe P, Soran A, et al. Axillary staging after neoadjuvant chemotherapy for breast cancer: a pilot study combining sentinel lymph node biopsy with radioactive seed localization of pre-treatment positive axillary lymph nodes. Ann Surg Oncol. 2016;23(5):1549-1553.

16. Kim E, Byon W, Lee K, et al. Feasibility of preoperative axillary lymph node marking with a clip in breast cancer patients before neoadjuvant chemotherapy: a preliminary study. World J Surg 2018;42(2):582-589.

17. Donker M, Straver ME, Wesseling J, et al. Marking axillary lymph nodes with radioactive iodine seeds for axillary staging after neoadjuvant systemic treatment in breast cancer patients: the MARI procedure. Ann Surg. 2015;261(2):378-382.

18. Siso C, de Torres J, Esqueva-Colmenarejo A, et al. Intraoperative ultrasound-guided excision of axillary clip in patients with nodepositive breast cancer treated with neoadjuvant therapy (ILINA Trial): a new tool to guide the excision of the clipped node after neoadjuvant treatment. Ann Surg Oncol. 2018;25(3):784-791.

19. Nguyen T, Heiken T, Glazebrook K, Boughey J. Localizing the clipped node in patients with node-positive breast cancer treated with neoadjuvant chemotherapy: early learning experience and challenges. Ann Surg Oncol. 2017;24(10):3011-3016.

20. van Nijnatten T, Simons J, Smidt M, et al. A novel less-invasive approach for axillary staging after neoadjuvant chemotherapy in patients with axillary node-positive breast cancer by combining radioactive iodine seed localization in the axilla with the sentinel node procedure (RISAS): a Dutch prospective multicenter validation study. Clin Breast Cancer. 2017;17(5):399-402.

21. Caudle A, Yang W, Krishnamurthy S, et al. Factors impacting the accuracy of targeted axillary dissection. 2017. www.surgonc.org. Accessed 6 June 2018. 